\title{
The incomplete Voronoi diagram and percolation analysis
}

\author{
L. Zaninetti ${ }^{1}$ \\ Istituto di Fisica Generale, Via Pietro Giuria 1, 10125 Turin, Italy
}

Received 27 September 1993; revised manuscript received 1 March 1994; accepted for publication 4 April 1994

Communicated by A.R. Bishop

\begin{abstract}
We first introduce the concept of incompleteness of the sides/edges in the 2D/3D Voronoi diagram introducing an appropriate probability; we then search for a percolation cluster and its relative threshold probability that is determined through a large number of statistically independent realizations.
\end{abstract}

\section{Introduction}

The hunting area of the Voronoi diagram, despite the first official book [1], and the many published papers ranging from the field of materials science to astrophysics, still has some holes.

A new connection could be that with percolation analysis and we recall that recently this mixture has been used in astrophysics to detect structures in 2D (in particular the ROSAT X-ray data) [2], and in chemistry to analyze models of liquid and quenched rubidium [3].

Here using an approximate algorithm previously developed [4] we first introduce the concept of incompleteness in 2D and 3D, meaning that we select only a fraction of the sides/edges rather than the whole. Our concept of incompleteness is a little bit different from the classical one (also named disorder in the molecular context) that considers a fraction of the seeds. We subsequently compute the threshold values that allow percolation from one side to the opposite one of our working box or square.

\section{The 2D case}

As usual we approximate the Voronoi diagram by a digital image introducing a lattice made by a pixels $\times$ pixels lattice that covers a square; we then extract the little squares that are at the same distance from two seeds and we avoid the effect of the boundaries inserting the seeds in a bigger area. All details are explained in Ref. [4], and a typical result is shown in Fig. 1a.

Our purpose is to eliminate some sides with a random process; this in order to introduce the concept of incompleteness. First of all we attach a label to each side in a progressive order; we then introduce the probability $p_{\mathrm{s}}$, ranging from 0 to 1 , that denotes the fraction of selected sides,

selected sides $=p_{\mathrm{s}} \times$ total sides ,

we therefore extract this last number of sides with a random process.

We now recall that the site percolation often uses a quadratic lattice in which we occupy at random a

\footnotetext{
' E-mail: zaninetti@to.infn.it.
} 


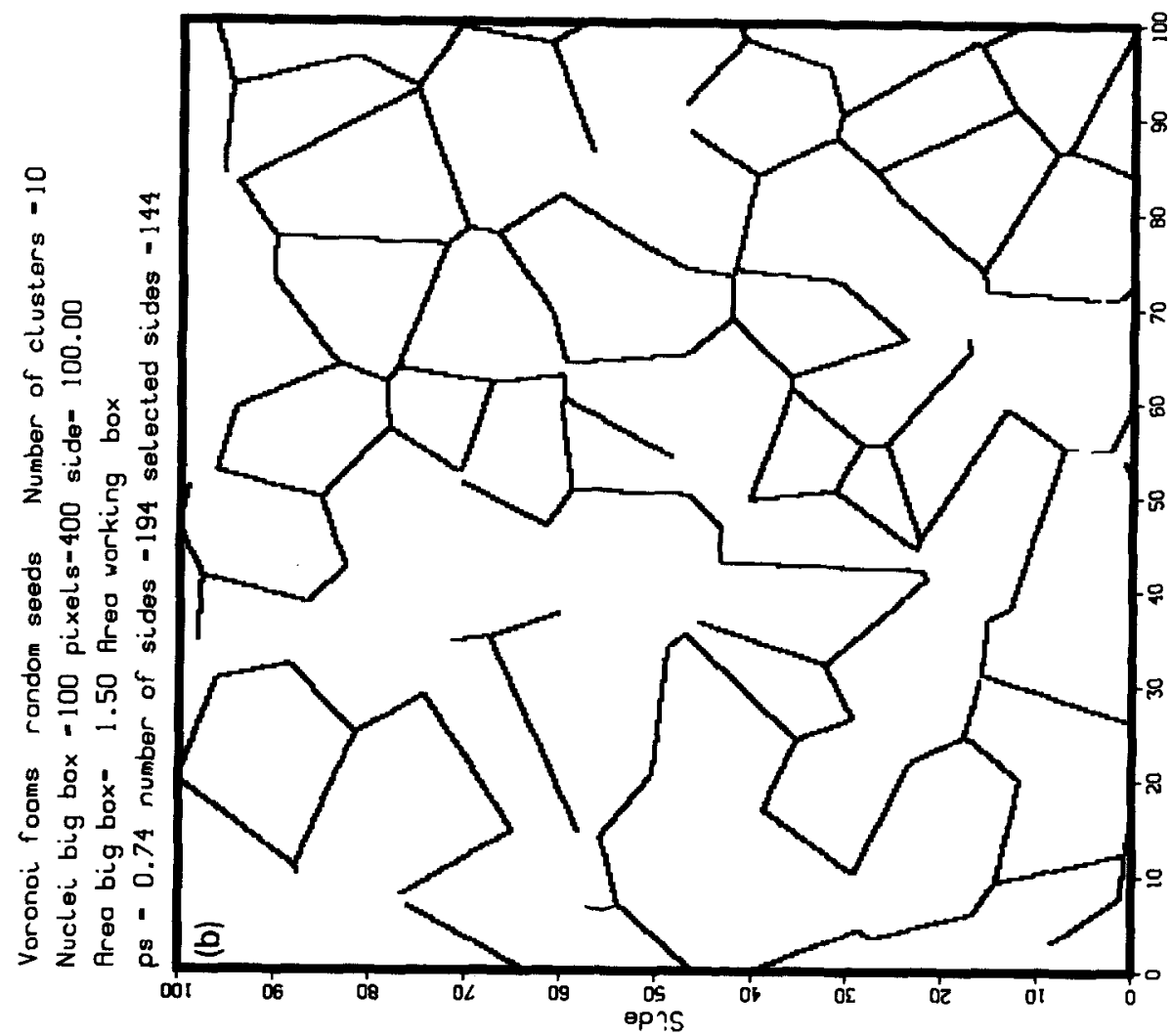

总

畧
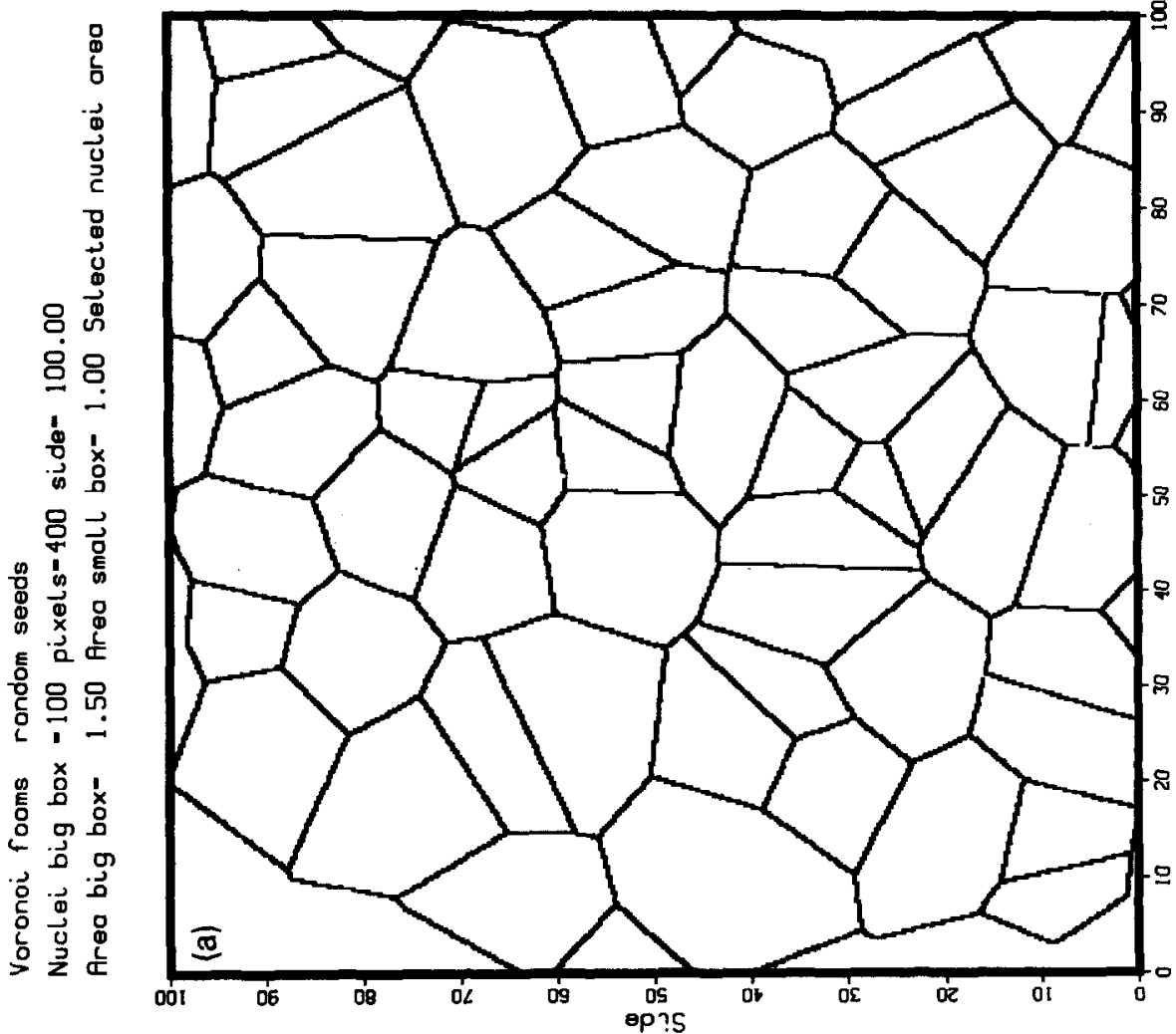


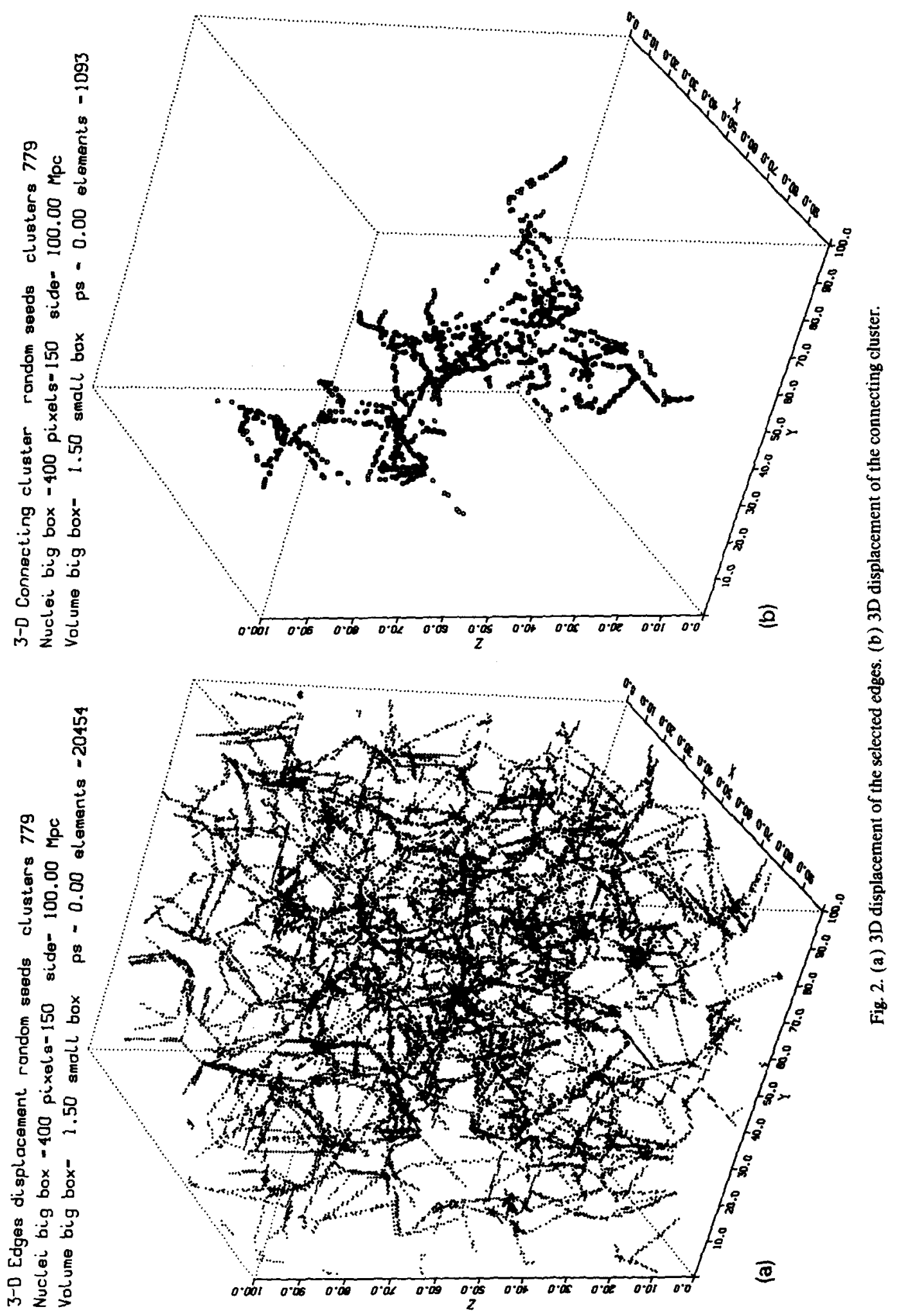


Table 1

The critical value of $p_{\mathrm{s}}$ for two different seed point distributions in $2 \mathrm{D}$ ( nuclei $=100$, pixels $=400$ )

\begin{tabular}{llll}
\hline Type of seeds & Experiments & $\bar{p}_{\mathrm{s}}$ & $s$ \\
\hline random & 20 & 0.70 & 0.07 \\
eigenvalues & 20 & 0.66 & 0.04 \\
\hline
\end{tabular}

fraction of the nodes of the square grid. Given a pixels $\times$ pixels lattice the percolation probability is defined as the probability to have a cluster connecting the left and right edges or the top and bottom edges: this probability turns out to be $\approx 0.6$.

We now adopt the point of view of percolation and we identify the two lattices, the points belonging to the Voronoi polygons now becoming the occupied sites; for practical purposes the sites consist of squares and the number of neighbors available for percolation is 8 .

We then recall that two sites belong to the same cluster if they are connected by a path of occupied sites; therefore for a given value of $p_{\mathrm{s}}$ we will have a certain number of clusters. The critical value of $p_{\mathrm{s}}$ now separates the phase of finite clusters of sites from a phase of an infinite cluster. In order to give a statistical meaning to $p_{\mathrm{s}}$ its value should be determined for a large number of statistically independent experiments. We remember that we generate the seeds through two different processes: the random number and the eigenvalue numbers, see Ref. [4]. In order to fix some points we made 20 different realizations of such a square and we therefore obtain an averaged value of $p_{\mathrm{s}}$ and a connected value of the standard deviation that are reported in Table 1 .

The network obtained in one of such a run is shown in Fig. $1 b$.

\section{The 3D case}

The basic lattice has now pixels $\times$ pixels $\times$ pixels elements that cover a cube of volume side ${ }^{3}$ and as usual in order to simulate the boundary conditions we insert the seeds in a bigger volume.

We make a $3 \mathrm{D}$ scanning of the box and we select only the pixels at the same distance from three nuclei and we label them on the base of the edge to which they belong. After these operations we select a certain
Table 2

The critical value of $p_{\mathrm{s}}$ for two different seed point distributions when the edges are considered in $3 D$ (nuclei $=400$, pixels $=150$ )

\begin{tabular}{llll}
\hline Type of secds & Experiments & $\overline{p_{\mathrm{s}}}$ & $s$ \\
\hline random & 10 & 0.62 & 0.04 \\
\hline
\end{tabular}

number of edges following the rule

selected edges $=p_{\mathrm{s}} \times$ total edges .

A typical network is shown in Fig. 2a. The cluster that connects two opposite faces of the cube is shown in Fig. $2 b$ and we remember that in this case the number of neighbors is 26 .

Also here we should apply statistical arguments in order to find the averaged value of $p_{\mathrm{s}}$, this time only one type of numbers (the random one) being available, see Table 2. Due to the great improvement in the computing facilities we were able to handle a $150 \times 150 \times 150$ cubic lattice.

\section{Conclusions}

We have built an algorithm able to select some of the sides/edges of the irregular polygons/polyhedra. Given some typical systems that present a phase transition like conductor-superconductor or magnet-paramagnet we have presented a new kind of lattice nearer to the molecular dynamic configuration than the classical one. Further on, identifying the selected pixels with the site position on a $2 \mathrm{D} / 3 \mathrm{D}$ regular percolation lattice, we can compute the threshold probabilities that turn out to be similar to the classical ones.

\section{References}

[1] A. Okabe, B. Boots and K. Sugihara, Spatial tessellations concepts and applications of Voronoi diagrams (Chichester, 1992).

[2] H. Ebeling and G, Wiedenmann, Phys. Rev. E 47 (1993) 704.

[3] N.N. Medvedev, A. Geiger and W. Brostow, J. Chem. Phys. 93 (1990) 8337.

[4] L. Zaninetti, Phys. Lett. A 165 (1992) 143. 